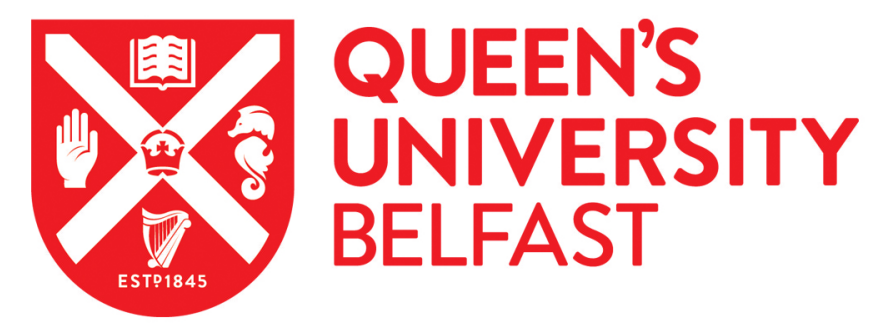

\title{
Bolivian health and social care professionals' experiences of decision- making in oncology and palliative care
}

Roulston, A., \& Haynes, T. (2015). Bolivian health and social care professionals' experiences of decision-making in oncology and palliative care. British Journal of Social Work, 45(4), 1277-1295.

https://doi.org/10.1093/bjsw/bct186

Published in:

British Journal of Social Work

Document Version:

Peer reviewed version

Queen's University Belfast - Research Portal:

Link to publication record in Queen's University Belfast Research Portal

Publisher rights

(c) 2014 The Author 2014 Published by Oxford University Press on behalf of The British Association of Social Workers. All rights reserved. This is a pre-copyedited, author-produced PDF of an article accepted for publication in the British Journal of Social Work following peer review. The version of recordis available online at: http://bjsw.oxfordjournals.org/content/45/4/1277

\section{General rights}

Copyright for the publications made accessible via the Queen's University Belfast Research Portal is retained by the author(s) and / or other copyright owners and it is a condition of accessing these publications that users recognise and abide by the legal requirements associated with these rights.

Take down policy

The Research Portal is Queen's institutional repository that provides access to Queen's research output. Every effort has been made to ensure that content in the Research Portal does not infringe any person's rights, or applicable UK laws. If you discover content in the Research Portal that you believe breaches copyright or violates any law, please contact openaccess@qub.ac.uk. 


\section{Abstract \\ Bolivian health and social care professionals' experiences of decision-making in oncology and palliative care}

In the United Kingdom, end-of-life care strategies recommend patients and families are involved in decision-making around treatment and care. In Bolivia, such strategies do not exist, and access to oncology services depends on finance, geography, education and culture. Compared to more developed countries, the delivery of oncology services in Latin America may result in a higher percentage of patients presenting with advanced incurable disease.

The objective of this study was to explore decision-making experiences of health and social care professionals who cared for oncology and palliative care patients attending the Instituto Oncológico Nacional, Cochabamba (Bolivia). Patients were predominantly from the Quechua tradition, which has its own ethnic diversity, linguistic distinctions, and economic systems. Qualitative data were collected during focus groups. Data analysis was conducted using Interpretative Phenomenological Analysis. Three interrelated themes emerged: (1) Making sense of structures of experience and relationality; (2) Frustration with the system; and (3) The challenges of promoting shared decision-making. The study uncovered participants' lived experiences, emotions and perceptions of providing care for Quechua patients. There was evidence of structural inequalities, the marginalisation of Quechua patients, and areas of concern that social workers might well be equipped to respond to, such as accessing finances for treatment/care, education and alleviating psychological or spiritual suffering.

Keywords: Oncology; palliative care; decision-making; Interpretative Phenomenological Analysis; Latin America

\section{Bolivian health and social care professionals' experiences of decision-making in oncology and palliative care}




\section{Introduction}

Oncology and palliative care teams traditionally consist of a relatively small nucleus of specialist health and social care professionals, to whom patients are referred for assessment and care planning. According to the World Health Organisation definition:

'Palliative care is an approach that improves the quality of life of patients and their families facing the problem associated with life-threatening illness, through the prevention and relief of suffering by means of early identification and impeccable assessment and treatment of pain and other problems, physical, psychosocial and spiritual' (WHO, 2002: 84).

Medical, nursing and allied health professionals are primarily responsible for relieving the physical symptoms of palliative care patients and improving their quality of life. However, social, emotional and spiritual problems, which can exacerbate physical symptoms, are normally explored by social workers and chaplains. Given the prevalence of loss and change experienced by palliative care patients, and the need for natural engagement with the patient's family, community and cultural identity, social workers need to be involved (Reith and Payne, 2009: 16). Traditionally, the palliative care social worker's role includes: enabling communication and family relationships; encouraging reflection; providing financial advice and information; liaising with colleagues to establish domiciliary services; supporting individuals with anxiety; encouraging self-determination; challenging discrimination; and conducting assessments (Sheldon, 2000: 493-496). As meeting the holistic care needs of patients and families can only be achieved when members of the multi-disciplinary team (MDT) 
work interdependently to achieve common goals (Speck, 2003), social workers are regarded as essential (Holloway and Moss, 2010). Partnership working between MDT members, patients and family carers is also key to ensuring a participatory, collaborative and well coordinated approach to shared decision-making (Orchard and Curran, 2003). In the UK, the government's ambition is to achieve healthcare outcomes that are among the best in the world. To achieve this, they want 'shared decision-making to become the norm', with all treatment decision-making being made in partnership between patients and physicians (DoH, 2010: 13; DHSSPSNI, 2011). This paper explores the range of barriers which may prevent such an ambitious approach to the uptake and delivery of oncology and palliative care services in Bolivia.

\section{Economic barriers}

In most countries, oncology and palliative care services are accessible and aim to reduce mortality rates and improve the quality of life of patients and their families. However, Bolivia is a developing country where an individual's average annual income is $\$ 911$ (compared to $\$ 25,000$ in the UK) and the structure of the health care system in Bolivia means that access to oncology and palliative care services is dependent on individuals paying privately. As a consequence, access to treatment is largely based on affordability, which naturally impacts on the patient's quality of life and life expectancy. Subsequently, the social work role is dominated by assisting patients to source finance to pay for their treatment and travel costs, rather than conducting assessments, facilitating communication or supporting patients and families with palliative or end-of-life care issues. When compared to more developed counties, other factors that may interfere with the uptake of oncology services in Latin American countries are: a lack of transportation and accommodation near the treatment centre; 
difficulties in following up patients who travel long distances; and non-functioning radiotherapy units due to mechanical breakdowns, which are expensive and timeconsuming to repair (Pavlovsky and Litvak, 1984).

\section{Cultural beliefs}

Approximately 10-11million indigenous Americans from the Quechua tradition reside in Bolivia and its bordering countries (Brazil, Paraguay, Argentina, Chile and Peru). The fact that Quechua people have their own ethnic, economic and linguistic distinctions may have an impact on the uptake of oncology and palliative care services. Whilst it might be expected that the impact of cancer produces similar human responses in people around the world, there is evidence that individual responses are affected by culture, myths and beliefs (Granda-Cameron, 1999). For example, an examination of ethical decision-making in palliative care highlighted that reluctance to share the truth with patients about their diagnosis and prognosis was frequently associated with cultural pressures, and a narrow understanding of autonomy (Taboada and Bruera, 2001). Granda-Cameron (1999) posited that common cultural health beliefs play a significant role in the way Latin Americans perceive the experience of cancer. Such perceptions can be compounded by limited awareness of early signs and symptoms of cancer on the part of patients, relatives and even doctors; the modality of treatment on offer (depending on which medical professional the patient sees first); and poor communication between professionals and patients (Pavlovsky and Litvak, 1984). Thus, those co-ordinating services should have a clear understanding of the needs, concerns and beliefs of local communities to ensure that services will be accessible, acceptable and utilised.

\section{Quality of care}


Vigil et al (2007) and Torres-Vigil et al (2008) examined professionals' experiences of the quality of care in advanced cancer, using a large sample of physicians and nurses from Argentina, Brazil, Cuba, Mexico, and Peru. Findings emphasised issues regarding access to care; affordability of treatment; low prioritisation of palliative care policies; and lack of education for physicians and nurses. It has also been reported that availability of opioid analgesics was insufficient for pain due to legislative, educational and policy issues in Latin America (De Lima et al., 1997; Bruera, 1993). In a more recent study, palliative care was reported to be a neglected area of health care provision in Argentina, with perceived neglect being exacerbated by the prevalence of chronic diseases; an aging population; prevalence of delayed diagnosis; and a lack of curative facilities (Wenk and Bertolino, 2007). Although little is known about how Latin Americans make hospice decisions, Kreling et al (2010) found that hospice use is low. Cultural values of denial, secrecy about prognosis and a collective, family-centred system influenced hospice decisions moreso in Latinos, when compared to non-Latinos (Kreling et al., 2010). This highlights a significant dilemma, such as how to explore hospice care with a patient and family from the Quechua tradition, who prefer not to discuss a terminal prognosis. In the context of hospice decision-making in Bolivia, further exploration is required of how information is channelled, blocked or used as it is unclear how interpersonal decisional transactions take place between patients, professionals and relatives (Llewellyn-Thomas, 1995) and whether or not shared decision-making is common practice.

\section{Communication}

Attitudes and beliefs of palliative care physicians regarding communication with the terminally ill in Europe, Latin America and Canada were examined by Bruera et al 
(2000). The findings suggested regional differences regarding communication with the palliative patient. Whilst Latin American physicians believed that patients should be aware of their diagnosis and the terminal nature of their illness, the findings revealed that for a substantial number of patients, this was not the case. Latin American physicians valued autonomy relatively less than justice and beneficence, as compared to Canadians. The authors suggested that this may reflect a paternalistic pattern of practice, or an increased concern about patients' financial status and their ability to access healthcare. Latin American physicians felt that only a minority of their patients wanted to know the terminal nature of their illness, and favoured patient-based decision-making less frequently when compared to their Canadian counterparts. This pattern of communication could reflect cultural differences which may also have existed within the regions investigated in our study. Communication between palliative care physicians and their patients regarding diagnosis and prognosis has not been extensively researched in the Bolivian context. Within the literature, we found a paucity of data on the nature of institutional cancer care specifically in Bolivia; the provision of psychosocial assistance by healthcare institutions; and the views of professionals in respect of the challenges they face with decision-making around oncology and palliative care.

\section{Methods}

This study aimed to explore the experiences of health and social care professionals who supported oncology and palliative care patients in Bolivia with healthcare decision-making. The main objectives of the study were to: 
- Identify the practical and ethical challenges in supporting patients with healthcare decision-making.

- Explore the political, cultural and economic factors that may influence decisionmaking in this field.

- Gain an understanding of the experiences of health and social care professionals who manage treatment and care decision-making in an oncology and palliative care setting.

\section{Design}

Qualitative data were obtained during three 45 minute focus group meetings, which were held with consenting medical, nursing, social work and physiotherapy professionals $(n=17)$, who represented the MDT. The research setting was the Instituto Oncológico Nacional in Cochabamba (Bolivia), which offers inpatient and outpatient oncology and community palliative care services. In the year prior to data collection (2009), care was provided to 874 patients (aged <64 years), predominantly from the Quechua tradition $(n=684,78 \%)$.

\section{Recruitment}

The Medical Director screened professionals against inclusion and exclusion criteria, and offered study information, prior to the lead author meeting interested staff. Electronic equipment was purchased to remunerate the team for time invested. Formal ethical approval was granted by Queen's University Belfast (A10/01). 
Version 23.07.13

\section{Data collection}

Focus groups allow multiple voices to be heard at one sitting, and enable the researcher to engage with a naturally occurring group (Palmer et al, 2010). A focus group methodology, rather than questionnaires or face-to-face interviews was selected, so that an existing MDT could interact and explore the experiential and professional perspectives of the challenges around decision-making in oncology and palliative care (Krueger and Casey, 2000). This methodology also provided valuable data from collective narratives (Payne, 2007). Initially, all participants completed a demographic questionnaire. The interview schedule, which was informed by the Llewellyn-Thomas (1995) framework, was used to collect focus group data. The focus group meetings, which were conducted in Spanish using a local interpreter, were audio-recorded; transcribed verbatim by a Bolivian typist; translated into English by a professional interpreter; and analysed by both authors. Participants were provided with a copy of the full transcript, which enabled them to verify the accuracy of the contents.

\section{Data analysis}

Relatively few studies have utilised IPA with a focus group methodology. This method encourages the researcher to look beyond the superficial explanation or description of an experience, and to understand human behaviours, actions and experiences, whilst maintaining the context of everyday lived experiences, where meaning resides (Robertson-Malt, 1999). Its theoretical foundations are in phenomenology, hermeneutics, and idiography (Smith et al., 2009). Our data analysis was guided by Palmer et al (2010), who advocate seven steps to be undertaken when identifying the emergent themes: identifying objects of concern and experiential claims; positionality of the researcher and participants; roles and relationships; organisations and systems; 
stories told by participants; language in terms of patterns, context and function; and adaptation of emergent themes. When undertaking data analysis, Koch and Harrington (1998) recommend that IPA researchers need to 'bracket' their taken-forgranted world and Palmer et al (2010) recommend researchers consider 'positionality'. In this analytical process, attention was drawn to the cultural differences and linguistic competences of the researcher (AR), the different health and social care system in her country of origin, and her professional experience. Both authors independently analysed the data to identify the objects of concern and emergent themes. Differences in interpretation were discussed and resolved by consensus. Quotes were selected that encapsulated the semantics of the emerging themes. In the preliminary stages of analysis, seven themes emerged: access and barriers to services; cultural and economic obstacles to screening, diagnosis and treatment; decision-making; institutional and socio-political limitations; patients' socio-demographic characteristics; professional coping; and interpersonal relationships between patient, professional and family. These preliminary themes were presented to the participants, during a followup visit to Bolivia (July 2011), which enabled them to confirm our interpretations. These were subsequently collapsed into three discrete but interrelated themes.

\section{Limitations}

This study was conducted with professionals in one Institute in Bolivia. Therefore, findings are not generalisable to other Latin American countries. Findings would have had more rigour if triangulated with the perspectives of patients and family carers. As this study was conducted during the lead author's first visit to Bolivia, she believed it was important to establish effective working relationships with professionals, prior to requesting contact with patients or family carers. The focus groups were conducted in 
Spanish, using an interpreter, which affected the researcher's ability to explore issues in greater depth. As with standard IPA, the authors monitored ways of mapping language used throughout the reverse-translated transcript onto emergent themes, with attention being paid to repetition, tone, emphasis and speech patterns. However, due to the linguistic differences, one limitation of this area of analysis was the inability to reliably discern euphemism, metaphor or idiom.

\section{Findings}

\section{Theme 1: Making sense of structures of experience and relationality}

The socio-demographic circumstances of patients, and how they impacted on decision-making, were a key object of concern. The participants identified a number of barriers associated with the diagnosis, treatment and care of patients with cancer, which included finance, culture, geography and professional suffering.

Finance

According to our findings, the key barrier preventing lower income families obtaining a cancer diagnosis was finance, as those 'families who live in marginal areas can't afford to find out whether they have a tumour or not, can they?' (ID9: Doctor). Professionals perceived that economic factors also affected decision-making around treatment options:

'We should take cultural and economic factors into consideration, because these have a huge influence particularly in our society and in the approach we should take when examining the patient's treatment options. [Pause] Many people do not have the economic resources to accept radiotherapy or 
chemotherapy treatment and that plays a huge role' (ID14: Palliative Care Consultant).

The following extract graphically illustrates how finances were a barrier to patients obtaining treatment and a challenge for the social worker employed at the Institute:

‘..from the moment they arrive, we ask them for different tests that require more or less about 1000 Bolivianos [\$150] [Pause] and for them that is a restriction...In many cases they decide to make the decision to abandon the treatment. Others decide to start campaigns to raise funds and ask some relatives or other people to help them...' (ID11: Social Worker).

Overall, participants acknowledged that patients' limited financial means were a barrier to obtaining a cancer diagnosis, treatment and care.

\section{Culture}

Participants identified cultural barriers preventing patients from the Quechua tradition accessing oncology services at the Institute.

'For a large percentage it could be economic reasons, but for an even bigger percentage, it's cultural, something to do with their education, because people are afraid to go to a health centre, afraid for all different types of reasons...or maybe in cases of abuse' (ID5: Oncologist).

It was suggested that Quechua women regarded medical procedures as invasive or potentially abusive, as they were outside their normal cultural experiences. This point was elaborated on by another respondent, who offered a gender specific explanation, 
which suggested that 'invasive examinations' required to diagnose or treat an illness

\section{may act as a barrier to accessing care:}

'People come from the rural area, and if they are women, they have never been to a doctor. They have given birth to their children at home, so the fact that they have to go to the doctor, and that doctor has to examine them, has to do an invasive examination, is obviously something very traumatic for them' (ID3: Radiotherapist).

One participant (ID5) suggested that introducing health education policies in schools and offering preventative control programmes may help, as 'prevention is better than treatment'.

Geography

The third barrier identified was geographical distance, which prevented 'income-poor patients from isolated provinces' from accessing palliative home care services (ID10: Doctor), and affected decision-making in relation to the uptake or continuation of cancer treatments. There was widespread acknowledgement that chemotherapy treatment patterns posed problems for patients from rural communities, who abandoned treatment due to the need to be closer to their home, family or employment.

'Treatments require fairly long periods of time that will take them away from their routine lives...they will have to be months away from home, from their work, their family and sometimes means that they abandon the treatments' (ID3: Radiotherapist). 
$\underline{\text { Research data offered the authors insight into the unique socio-demographic profile }}$ of patients from the Quechua culture attending the Oncology Institute. There was consensus within the focus group that Quechua people appear to be more accepting of the futility of their treatment and their impending death.

'Some patients...from the Quechua culture, when they hear that the treatment is not going to cure them, they analyse their economic income and the distance... and they accept that they're going to die' (ID10: Doctor).

In summary, participants felt that economic and geographical factors prompted decisions to cease treatment, and provoked feelings of helplessness and acceptance among patients.

Professional suffering

Professionals developed close working relationships with patients and their family, during their cancer journey.

'A personal relationship is formed between the health staff and the patient...If they are going to get better, we encourage them, and if not...we try to help them endure the situation without getting depressed...I personally think this doctorpatient relationship should always be like this, or even better, and we should create another psychology service for oncology patients (ID10: Doctor).

However, the following accounts illustrate how different oncology and palliative care professionals managed and coped with these working relationships. The radiotherapist (ID3) had a clearly defined coping strategy, whereby she established personal and professional 'boundaries'. 
'I think that as doctors, we learn to put up barriers. It's important to have boundaries, isn't it? I don't want to say that we de-humanise ourselves, but there has to be a boundary, where we know that our job ends here, and we close the door, so to speak' (ID3: Radiotherapist).

In contrast, the palliative care consultant (ID14) felt heavily involved in the 'suffering' experienced by patients and their families:

'...it is unfortunately difficult to put up that barrier, and we have to get involved with the family's suffering and the patient's suffering....we suffer with the family's pain' (ID14: Palliative Care Consultant).

This was echoed by the social worker, who experienced similar emotions, over time:

'It's obviously important not to lose sight of our humanitarian feelings, for any reason...it is inevitable to see the suffering...It is really hard to watch people losing weight, or seeing their physical condition change over time...and the family feels helpless, no matter how much money they have' (ID11: Social Worker).

Whilst promoting a 'good death' and offering high quality end-of-life care was important for the group, the individual emotional responses to the suffering of patients varied between participants.

\section{Theme 2: Frustration with the system}

Participants described their orientations towards their Institute, other organisations and referral systems, including the sorts of meanings, expectations and consequences they attributed to them. 


\section{Public awareness}

As illustrated in the following extract, there appeared to be a lack of public awareness of the Institute's existence, and a sense that they could be more proactive in providing more information:

'One thing is the lack of knowledge and marketing, we could say publicity for the Oncology Institute. I mean that [we are] outside the urban centre of Cochabamba and many people don't know about it...information coming from here is lacking' (ID9: Doctor).

Despite this lack of awareness, there was an emerging reality from the participants' experiences that seeking medical examinations was an alien and disturbing concept for some patients, and that treatment processes confused their habituated practices. As illustrated by one participant (ID4), 'a deficiency in society is screening and referral to specialist oncology services'. Participants believed that deficits in the education of the public and the training of professionals prevented early detection and resulted in unnecessary instances of advanced disease.

\section{Lack of information, education and training}

A lack of information on the patient's part and lack of training on the general professional's part were other areas of concern for one participant, who believed they led to inappropriate treatment processes and patients presenting with advanced disease.

'They [general Hospital in Cochabamba] have [oncology] services but they are not very well informed yet. They are run by general doctors; maybe one or two 
oncology specialists, but they do not have a complete service in a MDT' (ID4: Surgeon)

'The first contact they [patients] have is with general doctors who treat them and when they reach our Institution they arrive in more advanced stages or they have been handled wrongly, and that to some degree, interferes with the correct management' (ID4: Surgeon).

There was an almost fatalistic attitude among professionals regarding patients' experiences with oncology services, and regret that mismanagement and misinformation could result in patients receiving inappropriate treatments and false hopes about cure, or result in the premature death of a patient. A more detailed examination of this participant's story revealed assumptions of an embodied and relational knowledge of patients; compassion when reflecting on misinformation; apportioning blame for mismanagement outside the Institution; and avoiding blame from within his Institution. One social worker suggested how educating people from different ethnic and socio-economic groups about cancer prevention and treatment, may help overcome instances of maltreatment or misinformation:

'I think it's very important to work with people from all different types of groups...so that people can understand that there are ways of preventing the disease, forms of treatment and explain to people what's done and what's not done at any given time, because obviously they are unaware of that and often they are misinformed, even by professionals (ID11: Social Worker).

In response to these perceived deficiencies, the medical, nursing and social work staff from the Institute co-facilitated bi-annual education programmes around prevention 
and early diagnosis, which created opportunities to offer more information to the public.

\section{Treatment}

Participants indicated the challenges in ensuring that the financial deficit between the income and the expenditure of running the Institute did not compromise the quality of care offered and or the maintenance of equipment required for treatment.

To promote choice, cheaper treatments were explored with patients, despite professionals' knowledge that they were potentially more toxic or less effective.

'We tell them that they have alternatives, depending on their economic ability to pay for the medicines... and we have to explain because the patient does not know that he or she has a cheaper option... which usually have higher toxicity levels and, in many cases, are less effective, but can help a lot in many cases' (ID5: Oncologist).

However, offering less effective treatment raised an ethical predicament in clinical practice. A general lack of information and education resulted in difficulties for the radiotherapist when explaining treatment processes to patients:

'There are many patients, and even doctors who think the first option is surgery and the patients themselves believe that if they have a tumour and it's removed then they are cured and that surgery would always be the best option. So to convince them about radiotherapy, it's difficult to explain to them how it works, in a way they'll understand, how lying down under the machine will resolve their tumour. It's hard for them to accept this treatment, and I think it's the same with chemotherapy' (ID3: Radiotherapist). 
Participants expressed frustration at the Institute's limited access to effective treatments, and inability to legally participate in research trials. It was suggested that 'lobbying the government to authorise research studies' would help alleviate the situation (ID3: Radiotherapist).

Psychological support

There was recognition of the value of the support services currently offered to patients, carers and professionals, but there was a sense that other psychosocial services, such as bereavement support, counselling and spiritual care services were lacking. Although social workers informally offered patients psychological support, there was a sense that perceived stigma would result in patients rejecting formal psychology services:

‘...only very few patients accept this support... They see it like going to a psychiatrist and how would I think about going to a psychiatrist, no?...In the past, it looked bad if someone took their child to the psychologist and it even looks bad for an oncology patient to ask for psychological support' (ID3: Radiotherapist).

Overall, there was a sense of resignation, regret and acceptance that rejecting psychological support may have become embedded in practice, despite professionals advocating the benefits of developing services.

\section{Theme 3: The challenges of promoting shared decision-making}

The participants highlighted certain aspects of interactions throughout the decisionmaking process, including communication, the patient's loss of autonomy and the ethical predicaments surrounding euthanasia. 


\section{Communication}

Communication with patients about their diagnosis, treatment options and anticipated prognosis was an important object of concern for participants. In some cases, the dominance of family members resulted in requests to collude and block such information from the patient. Participants highlighted that when these aspects of a healthcare decision were not fully presented to the patient, perspectives from each member of the triad (professionals, patient and family) on preferences and expectations around end-of-life care became distorted. The subsequent ethical dilemma and ambivalence among professionals was apparent, particularly when parents wanted to protect their children from potentially devastating news:

'The same thing happens when dealing with young people and the parents come, and they come with the attitude of wanting to protect their children. They don't want the patient to find out about the diagnosis, especially when it's a terminal illness' (ID3: Radiotherapist).

However, to avoid collusion, some professionals advocated gradual information sharing, appropriate to the patient's capacity, health state and engagement, which was indicative of a 'deliberative model' of the patient-professional decision-making process (Emanuel and Emanuel, 1992).

'We have to explain to the family that it's important for the patient to know what he has, because they are going to receive treatments...so the patient has to be aware, and being aware together with the team and the family in the fight may improve his chances at living and improve his quality of life. So we explain to the family the need for the patient to be prepared to hear about his diagnosis 
progressively and gradually, according to his or her capacity... and the questions a patient asks are usually the question he or she is prepared to hear the answer to' (ID5: Oncologist).

As illustrated above, physicians believed that being open with patients ensured that they could choose to be involved in the 'fight' against cancer, which may improve their chances of survival and quality of life.

Whilst professionals recognised that some patients may be in denial of their situation, preferring not to ask questions, they believed that the large majority are fully aware 'that they have a problem that's threatening their life' (ID4: Surgeon). This view was echoed by another respondent who stated: 'They know they are dying, but they are just there, stoically and without asking any questions, or maybe they don't want to know and simply don't ask' (ID3: Radiotherapist). There was also recognition that not all patients wish to discuss their impending death, with some adopting a more passive stance.

'The large majority of patients prefer not to talk about their death...It is usually the family that talks about the patient's death, about how long, when, how, etc. The patient usually avoids it and has a more passive attitude' (ID5: Oncologist).

Some participants experienced regret with the patient's preference not to discuss death, and felt a sense of loss at the patient's apparent sacrifice of a future temporal dimension or the opportunity to attend to unfinished business or reflect on their life. In relation to preferred place of care, participants held the belief that most patients prefer to die at home, yet, professionals usually initiated discussions with the family...as 'the final decision is assumed by the family' (ID14: Palliative Care Consultant). 


\section{Euthanasia}

Despite being illegal in Bolivia, euthanasia raised an ethical predicament for the participants:

'Legally euthanasia is not accepted in Bolivia, but there have been patients who have requested it... They are usually well-educated people, with a higher cultural level, perhaps with more money, but what they don't want is to deal with pain and suffering' (ID3: Radiotherapist).

This participant indicated that she personally endorsed euthanasia and respected patients who requested it: 'I personally accept euthanasia, I mean I very much respect the patient who asks for euthanasia, but unfortunately we can't help them' (ID3: Radiotherapist). In addition, professionals observed that 'young people who are normally productive, people who don't want to be a burden for their parents and want to act conscientiously' were also more likely to request euthanasia (ID4: Surgeon). One respondent found euthanasia requests challenging, both personally and professionally, and relied on her communication skills to offer psychological comfort and spiritual guidance in the patient's hour of need:

'Often the patients ask us...if there is any way of speeding things up. 'Help us, Miss' they say, 'I want to die now! I don't want to put up with any more'... The only thing we can do is give them psychological support, and explain to them that the illness just has to follow its course and that it's not in our hands, but that it depends on a god, no?' (ID8: Nurse). 
Whilst some participants expressed personal views supporting euthanasia, this aspect of shared decision-making was constrained by Bolivian law, at the time of this study.

\section{Discussion}

Analysis uncovered the essence of shared experiences and a deeper understanding of the plight of Quechua people, which elicited a range of emotions from participants including remorse, sadness, exasperation, despair and helplessness. The detailed emotive and personal accounts of their professional caring experiences and their difficulty coping are illustrative of the impact of their lived experiences upon their sense of self. This went beyond the mainly superficial, descriptive and quantitative data revealed in the literature review, and enabled us to uncover and learn from the everyday lived experiences of what it is like to 'be' at the Institute, and how professionals attached meaning to their experiences. For example, the participants, who were an existing MDT, generally presented a united front, but at a deeper level, minor tensions surfaced around professional roles and coping behaviours. Findings revealed the inevitable development of interpersonal relationships between palliative care professionals, patients and families. This attachment appeared to be more challenging for those professionals who were involved in the 'suffering' of the patient and family, compared to those who focused on treatment and employed self-protecting barriers.

Our findings supported existing literature, which indicated that uptake and availability of high quality cancer services in Latin American countries was often prevented or 
delayed due to barriers including economics, education (Vigil et al., 2007; Torres-Vigil et al., 2008), geography (Pavolvsky and Litvak, 1984), who the patient saw first (Pavolvsky and Litvak, 1984); and common cultural beliefs (Granda-Cameron, 1999; Dein, 2006; Kreling et al., 2010).

Although economic deficiencies in health and social care are reported worldwide, participants experienced frustration with the infrastructure in Bolivia, where patients' economic circumstances influenced treatment and care on offer. As a team they struggled to balance the expenditure of providing high quality oncology services against how much patients could afford to pay for their care. It was assumed that when Quechua patients abandoned treatment it showed acceptance of their health state rather than their inability to secure financial aid.

Additionally, participants were anxious to educate people about the availability of specialist oncology services, alleviate general fears about cancer and prevent false hopes of cure. Whilst participants accepted that cultural barriers prevented many Quechua women from accepting medical examinations, there was a drive to educate people from rural communities about the benefits of cancer screening, which was similar to the findings of Agurto et al (2004).

Dein's proposed model of health education (2006), highlights measures which may overcome some of the barriers emphasised in the findings of this study, such as increasing the number of female physicians working within communities to promote the modesty of female patients; focusing health education on the family, rather than the individual; and communicating that if cancer is detected or treated early, the outcome may be more positive. 
How information and communication were channelled or blocked by individual members of the triad, raised ethical challenges for the professionals, as this compromised the patient's autonomy, independence and control over decision-making processes. For example, when professionals were asked to collude with overprotective families and withhold information from the patient, this contradicted their preference for a 'deliberative model' of the physician-patient relationship (Emanuel and Emanuel, 1992), which advocates shared decision-making, good communication and respect for unique socio-demographic characteristics. Our findings support those of Toboada and Bruera (2001), whereby physicians believed that patients should be aware of their diagnosis and prognosis, yet they respected some cultural preferences not to discuss dying.

Although illegal in Bolivia, participants revealed that well-educated and younger patients still requested euthanasia to avoid suffering or to alleviate the burden on their relatives. Participants from a range of disciplines expressed the challenges in dealing with euthanasia requests, and shared their experiences of offering support to patients in their hour of need. Although many ethical issues in palliative care are primarily medical, the debate about euthanasia is also relevant to social work (Reith and Payne, 2009). Finding meaning and experiencing peace and reconciliation in life and death features as a central spiritual concern for patients, and is one with which social work is very familiar (Holloway and Moss, 2010: 48). During conversations with the lead author, the Quechua social worker acknowledged that her role at the Institute was based on a traditional model of social case work (Horner, 2003), which focused on obtaining financial aid to support patients accessing treatment. Rather than being overwhelmed by the financial implications of living with cancer, social workers are well 
placed to work alongside medical and nursing staff to educate people about cancer screening, they have a key role to play within the delivery of palliative care services (Sheldon, 2000) and they are naturally trained to explore the family, community and cultural perspectives associated with cancer (Reith and Payne, 2009).

Participation in the focus group meetings offered scope to review how the social worker could work alongside members of the MDT to develop and deliver a more holistic menu of services to meet the wider psychosocial needs of the Quecha culture. Suggested improvements to practice included: involving the social worker in local education road-shows about cancer prevention, screening and treatment programmes; advocating for improved communication between individuals in families and local communities about their experiences of living with cancer; encouraging more involvement in decision-making around treatment and care; increasing understanding of the benefits of psychological support services; and adopting a more communitybased approach to overcome financial and geographical barriers to accessing care.

\section{Conclusion}

This study supports the proposition that there are ethical, clinical, socio-demographic and political reasons for exploring healthcare decision-making within oncology and palliative care in Latin America. Findings reinforce the economic and educational factors affecting treatment decision-making, and why professionals, including social workers, need to advocate for timely and honest communication with patients about treatment and preferences for care, rather than acquiescing to family requests for 
collusion. However, the need to respect the family's role in decision-making around different aspects of care must also be considered. Our participants frequently encountered real-life issues associated with helping patients and families to make choices that involved conflicting treatment options and palliative care decisions. Despite the ethical imperative to promote patient autonomy, the professionals were faced with several barriers which limited patient participation in decision-making. Whilst intrapersonal phenomena are not discrete entities, but interact with each other in complex ways, this study has shown how information can be channelled, blocked and used by the patient, professional and family in relation to decision-making. In addition, the findings offer us insight into the motivations and assumptions that these professionals attribute to the patient and family in terms of their expectations regarding treatment and care. This, in turn, affects the processes and outcomes of decisionmaking about health and social care at the Institute. These insights highlight the structural inequalities faced by marginalised Quechua patients and the importance of exploring and addressing the unique socio-demographic, economic, cultural, educational and geographical barriers in relation to cancer screening, treatment and care services in Bolivia. Findings emphasise a potential role for social workers in assisting patients to source finance for the cost of their treatment, care and transport; facilitate communication between patients, families and professionals, and offer emotional support and empathy to alleviate the psychological suffering of patients and their families. 


\section{References}

Agurto, I., Bishop, A., Sanchez, G., Betancourt, Z. and Robles, S. (2004) ‘Perceived barriers and benefits to cervical cancer screening in Latin America', Preventive Medicine, 39(1), pp. 91-98.

Bingham, A., Bishop, A., Coffey, P., Winkler, J., Bradley, J., Dzuba, I. and Agurto, I. (2003) 'Factors affecting utilization of cervical cancer prevention services in lowresource settings', Salud Publica de Mexico, 45 (Suppl 3), pp. S408-416.

Bradley, J., Barone, M., Mahe, C., Lewis, R. and Luciani, S. (2005) 'Delivering cervical cancer prevention services in low-resource settings', International Journal of Gynaecological Obstetrics, 89 (Suppl 2), pp. S21-29.

Bruera, E. (1993) 'Palliative care in Latin America', Journal of Pain and Symptom Management, 8(6), pp. 365-368.

Bruera, E., Neumann, C.M., Mazzocato, C., Stiefel, F. and Sala, R. (2000) 'Attitudes and beliefs of palliative care physicians regarding communication with terminally ill cancer patients', Palliative Medicine, 14 (4), pp. 287-298.

De Lima, L., Bruera, E., Joranson D.E., Guillermo, V., Cepeda, S., Quesada, L., Wenk, R., Pavajeau, M.C., Derio, L., Montejo, G., Castillo, G., Ruiz, F., Pupo, A.R., Caries, B., Paredes, E. and Schoeller, T. (1997) 'Opioid availability in Latin America: the Santo Domingo report. Progress since the Declaration of Florianópolis', Journal of Pain and Symptom Management, 13 (4), pp. 213-219.

Dein, S. (2006) Culture and Cancer Care. Anthropological Insights in Oncology. Berkshire: Open University Press. 
Emanuel, E.J. and Emanuel, L.L. (1992) 'Four models of the physician-patient relationship', JAMA, 256 (16), pp. 2221-2226.

Granda-Cameron, C. (1999) 'The experience of having cancer in Latin America', Cancer Nursing, 22 (1), pp. 51-57.

Holloway, M. and Moss, B. (2010) Spirituality and Social Work, Basingstoke: Palgrave Macmillan.

Horner, N. (2003) What is Social Work? Context and Perspectives. Exeter: Learning Matters.

Koch, T. and Harrington, A. (1998) 'Reconceptualizing rigour: the case for reflexivity', Journal of Advanced Nursing, 28 (4), pp. 882-890.

Kreling, B., Selsky, C., Perret-Gentil, M., Huerta, E. and Mandelblatt, J.S. 'Coalition Latin American Cancer Research (2010). 'The worst thing about hospice is that they talk about death': contrasting decisions and experience among immigrant Central South American Latinos with US-born White, non-Latino cancer caregivers' Palliative Medicine, 24 (4), pp. 427-434.

Krueger, R.A. and Casey, M.A. (2000) Focus Groups: A practical guide for applied research, (4 $4^{\text {th }}$ edition), London: Sage Publications.

Llewellyn-Thomas, H.A. (1995) 'Patients' health-care decision-making: A framework for descriptive and experimental investigations' Medical Decision Making, 5(2), pp. 101-106. 
Orchard, C.A. and Curran, V. (2003) Centres for Excellence for Interdisciplinary Collaborative Professional Practice. Prepared for the Office of Nursing Policy: Health Canada, Government of Canada.

Palmer, M., Larkin, M., de Visser, R. and Fadden, G. (2010) ‘Developing an Interpretative Phenomenological Approach to Focus Group data', Qualitative Research in Psychology, 7, pp. 99-121.

Pavlovsky, S. and Litvak, J. (1984) 'Multi-disciplinary consideration of cancer therapy in Latin America', International Journal of Radiation Oncology, Biology, Physics, 10 (Suppl 1), pp. 77-79.

Payne, S. (2007) 'Qualitative methods of data collection and analysis' in AddingtonHall, J.M., Bruera, E., Higginson, I.J. and Payne, S. (eds), Research Methods in Palliative Care, Oxford, Oxford University Press, pp. 139-161.

Reith, M. and Payne, M. (2009) Social work in end-of-life and palliative care, Bristol: Policy Press.

Reyes-Ortiz, C.A., Camacho, M.E., Amador, L.F., Velez, L.F., Ottenbacher, K.J. and Markides, K.S. (2007) 'The impact of education and literacy levels on cancer screening among older Latin American and Carribean adults', Cancer Control, 14 (4), pp. 388-395.

Roberston-Malt, S. (1999) 'Listening to them and reading me: a hermeneutic approach to understanding the experience of illness', Journal of Advanced Nursing, 29 (2), pp. 290-297. 
Sheldon, F. M. (2000) 'Dimensions of the role of the social workers in palliative care' Palliative Medicine, 14, pp. 491-498.

Smith, J.A., Flowers, P. and Larkin, M. (2009) Interpretative Phenomenological Analysis: Theory, method and research, London, Sage Publications.

Speck, P. (2009) Teamwork in Palliative Care: Fulfilling or Frustrating? Oxford, Oxford University Press.

Taboada, P. and Bruera, E. (2001) 'Ethical decision-making on communication in palliative cancer care: a personalist approach', Support Care Cancer, 9(5), pp. 335343.

Torres-Vigil, I., Aday, L.A., Reyes-Gibby, C., De Lima, L., Herrera, A.P., Mendoza, T. and Cleeland, C.S. (2008) 'Health care providers' assessments of the quality of advanced-cancer in Latin American medical institutions: a comparison of predictors in five countries: Argentin, Brazil, Cuba, Mexico and Peru', Journal of Pain and Palliative Care Pharmacotherapy, 22 (1), pp. 7-20.

Vigil, T., Aday, L.A., De Lima, L. and Cleeland, C.S. (2007) 'What predicts the quality of advanced cancer in Latin America? A look at five countries: Argentina, Brazil, Cuba, Mexico and Peru', Journal of Pain and Symptom Management, 34 (4), pp.315327.

Wenk, R. and Bertolino, M. (2007) 'Palliative Development in South America: a focus on Argentina', Journal of Pain and Symptom Management, 33 (5), pp. 645-650.

Winkler, J.L., Wittet, S., Bartolini, R.M., Creed-Kanashiro, H.M., Lazcano-Ponce, E., Lewis-Bell, M.J. and Penny, M.El. (2008) 'Determinants of human papillomavirus 
vaccine acceptability in Latin America and the Caribbean', Vaccine, 26 (Suppl 11): 73-79.

World Health Organisation (2002) National Cancer Control Programmes: Policies and Managerial Guidelines, (2 ${ }^{\text {nd }}$ edition), Geneva: WHO.

World Health Organisation (2012) Available online at http://www.who.int/cancer/detection/en/ (accessed 29th July 2012 